CASE REPORT

\title{
Massive pleural effusion and marked increase of CA-125
}

\author{
S F Hussain, J Grayez, A Grigorian, J T Green
}

Postgrad Med J 2004;80:300-301. doi: 10.1136/pgmj.2003.012377

The tumour associated CA-125 antigen is widely used in monitoring ovarian carcinoma. In women with a massive pleural effusion and ascites, markedly increased CA-125 levels may lead to an erroneous diagnosis of ovarian cancer. Very high levels of tumour markers may be present in patients with benign pleural effusion, ascites, and chronic liver disease. Raised levels of tumour markers in serum or pleural fluid, in the absence of positive cytology, should be interpreted with caution.

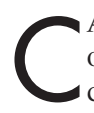
A-125 is a high molecular mass glycoprotein expressed on the cell surface of some derivatives of embryonic coelomic epithelium. The tumour associated CA-125 antigen is widely used in monitoring ovarian carcinoma. Serum CA-125 levels are increased in ovarian cancer and decrease after effective treatment. Massive pleural effusion is often malignant in nature. It had been suggested that CA- 125 titres (above $1000 \mathrm{U} / \mathrm{ml}$ ) could differentiate benign from malignant conditions. ${ }^{1}$ We report the case of a woman with massive pleural effusion, ascites, weight loss, and a marked increase of serum CA-125 titres. The implications of CA-125 measurement in this case scenario are discussed.

\section{CASE REPORT}

A 44 year old woman was admitted with two month history of progressive dyspnoea, which had failed to improve with antibiotics. She was a smoker of 25 pack-years and had consumed alcohol in excess of 30 units a week for several years. Her sister had breast cancer. Examination revealed jaundice with stigmata of chronic liver disease, moderate right sided pleural effusion, and gross ascites. There were no breast masses or lymphadenopathy. Pleural fluid analysis revealed a transudate with normal glucose and amylase levels; scanty mesothelial cells, no malignant cells; and negative culture for bacteria and tuberculosis. Blood results showed increased mean corpuscular volume (102 fl) and deranged liver function tests (total bilirubin $97 \mu \mathrm{mol} / \mathrm{l}$, aspartate aminotransferase $89 \mathrm{IU} / \mathrm{l}, \gamma$-glutamyltransferase $592 \mathrm{IU} / \mathrm{l}$, alkaline phosphatase $433 \mathrm{IU} / \mathrm{l}$, albumin $25 \mathrm{~g} / \mathrm{l}$, globulin $49 \mathrm{~g} / \mathrm{l}$, and international normalised ratio 1.6). Serum iron studies, serum viral hepatitis screen, $\alpha_{1}$-antitrypsin, caeruloplasmin, antinuclear, antineutrophil cytoplasmic antibody, and liver autoantibodies were all within the normal range. An ultrasound scan of the abdomen showed cirrhosis with gross ascites. Cultures and cytology of repeated ascitic fluid samples were negative. Serum CA-125 was markedly increased $(1987 \mathrm{U} / \mathrm{ml})$ with normal serum carcinoembryonic antigen, CA 19-9, and $\alpha$-fetoprotein.

Three days after admission a massive increase in the size of the pleural effusion was associated with dyspnoea and marked hypoxia that required pleural fluid drainage. There was a reluctance to accept the patient on the intensive care ward in view of the markedly raised CA-125 level, family history of breast cancer, and marked pleural effusion and ascites despite negative fluid cytology. Computed tomography revealed no evidence of chest, abdominal or pelvic malignancy, and demonstrated cirrhotic nodules in the liver, normal ovaries, and bilateral areas of pneumonic consolidation consistent with an infective process. The patient was mechanically ventilated for a week, treated with broad spectrum antibiotics and diuretics, and pleural fluid drained through an intercostal tube. Four weeks later her jaundice had improved, ascites and pleural effusion had resolved, and serum CA-125 level had decreased to $393 \mathrm{U} / \mathrm{ml}$. Two weeks later the patient developed nosocomial sepsis with hepatorenal syndrome and died. The relatives declined a postmortem examination.

\section{DISCUSSION}

Our patient had massive pleural effusion, ascites, and weight loss and was suspected to have an underlying malignancy based on high CA-125 titres. There was hesitation in offering intensive care treatment for her worsening respiratory failure. Subsequent investigations suggested cirrhosis with a negative liver screen; possibly alcohol induced cirrhosis. In a female patient, metastatic ovarian cancer may have a similar presentation and appropriate radiological and histological investigations, rather than tumour markers alone, should establish the diagnosis.

In a study of 328 patients, a CA-125 titre higher than $1000 \mathrm{U} / \mathrm{ml}$ was always due to the presence of cancer. ${ }^{1}$ Markedly raised serum and fluid CA-125 titres have been reported in benign conditions ${ }^{2}$ and represent production of the antigen in benign proliferating mesothelial cells. Increased serum CA-125 levels were detected in 52\% of patients with hepatic diseases, in $100 \%$ of patients with nongynaecological peritoneal carcinomatosis, and in $87 \%$ of patients with pleural effusion. ${ }^{3}$ Our patient had a combination of liver disease, ascites, and pleural effusion and this could have resulted in markedly increased CA-125 levels. Because of the high frequency of false positive results associated with many benign conditions, CA-125 is of little value as a screening test for ovarian carcinoma.

Pleural effusion with increased CA-125 levels may occur in pelvic conditions other than ovarian carcinoma. These include Meigs' syndrome (secondary to ovarian fibroma) ${ }^{4}$ and pseudo-Meigs' syndromes (secondary to other benign pelvic tumours). ${ }^{5}$ Removal of tumour is associated with a rapid decline in tumour markers. Increased CA-125 levels may occur with non-gynaecological malignancy such as lung cancer (69\% with metastatic disease), mediastinal teratoma, and non-Hodgkin's lymphoma. ${ }^{6}$ Tuberculosis is another cause of massive pleural effusion associated with increased levels of CA-125. ${ }^{7}$ Increased CA-125 levels may occur in connective tissue diseases, chronic constrictive pericarditis, and in patients on haemodialysis with pleural effusion. ${ }^{8}$

Pleural effusions are found in about $6 \%$ of patients with cirrhosis; two thirds of these are right sided. A large effusion in a cirrhotic, where there is no other explanation for its accumulation, is called a hepatic hydrothorax. It appears to form because of the movement of fluid from the abdomen through right sided diaphragmatic defects. Treatment is 
difficult as in many patients it is resistant to diuretics and dietary sodium restriction. Thoracocentesis usually leads to rapid reaccumulation of the effusion and a chest drain may be difficult to remove. ${ }^{9}$ Further options for treatment of a hepatic hydrothorax include surgical repair of the diaphragmatic defects, a transjugular intrahepatic portosystemic shunt (TIPS), or liver transplantation if indicated.

The major limitation of our case report was the absence of postmortem proof that there was no ovarian cancer. However, the presence of an underlying ovarian cancer was judged to be extremely unlikely in view of the negative ultrasound and negative computed tomography findings, negative cytology of repeated ascitic and pleural and fluid samples, and the improvement of serum CA-125 levels without any form of cancer treatment.

The recommendation that routine testing of tumour markers in pleural fluid greatly increases diagnostic effectiveness and avoids the need for invasive diagnostic tests ${ }^{10}$ is not supported by our case report. Increased levels of tumour markers in pleural fluid, in the absence of positive cytology, should be interpreted with caution.

\section{Authors' affiliations}

S F Hussain, J Grayez, A Grigorian, J T Green, Llandough Hospital, Penarth, Vale of Glamorgan, UK

Correspondence to: Dr Syed Fayyaz Hussain, Section of Pulmonary Medicine, Aga Khan University Hospital, Stadium Road, PO Box 3500,
Karachi 74800, Pakistan; sfh_pulmonary@yahoo.co.uk; fhussain@ akunet.org

Submitted 9 July 2003

Accepted 19 August 2003

\section{REFERENCES}

1 Le Thi Huoung D, Mohattane H, Piette JC, et al. Specificity of CA 125 tumor marker. A study of 328 cases of internal medicine. Presse Med 1988;17(43):2287-91.

2 Daoud E, Bodor G. CA-125 concentrations in malignant and nonmalignant disease. Clin Chem 1991;37:1968-74.

3 Topalak O, Saygili U, Soyturk $M$, et al. Serum, pleural effusion, and ascites CA-125 levels in ovarian cancer and nonovarian benign and malignant disease: a comparative study. Gynecol Oncol 2002;85:108-13.

4 Timmerman D, Moerman P, Vergote I. Meigs' syndrome with elevated serum CA 125 levels: two case reports and review of the literature. Gynecol Oncol 1995;59:405-8.

5 Ramondetta LM, Carlson JA Jr, Schwarting R. Atypical Meigs' syndrome and bilateral ovarian stromal hyperplasia. A case report. J Reprod Med 1997;42:603-5

6 Molina R, Filella X, Jo J, et al. CA 125 in biological fluids. Int J Biol Markers 1998;13:224-30.

7 Mansour M, Linden ER, Colby S, et al. Elevation of carcinoembryonic antigen and CA-125 in a patient with multivisceral tuberculosis. J Natl Med Assoc 1997;89:142-3.

8 Funaunchi M, Ikoma S, Yu H, et al. A case of progressive systemic sclerosis complicated by massive pleural effusion with elevated CA125. Lupus 2000;9:382-5.

9 Borchardt J, Smirnov A, Metchnik L, et al. Treating hepatic hydrothorax. BMJ 2003;326:751-2.

10 San Jose ME, Alvarez D, Valdes L, et al. Utility of tumour markers in the diagnosis of neoplastic pleural effusion. Clin Chim Acta 1997;265:193-205. 